\title{
Two-Loop Corrections to Top-Antitop Production at Hadron Colliders
}

\section{R. Bonciani*}

Laboratoire de Physique Subatomique et de Cosmologie, Université Joseph Fourier/CNRS-IN2P3/INPG, F-38026 Grenoble, France

E-mail: bonciani@lpsc.in2p3. fr

\section{A. Ferroglia}

New York City College of Technology 300 Jay Street, NY 11201 Brooklyn, USA

Email: AFerroglia@citytech.cuny.edu

\section{T. Gehrmann}

Institut für Theoretische Physik, Universität Zürich, CH-8057 Zürich, Switzerland

Email: Thomas.Gehrmann@uzh.ch

\section{A. von Manteuffel}

Institut für Theoretische Physik, Universität Zürich, CH-8057 Zurich, Switzerland

Email: manteuffel@physik.uzh.ch

\section{Studerus}

Fakultät für Physik Universität Bielefeld, D-33501 Bielefeld, Germany

Email: cedricephysik. uni-bielefeld.de

The status of the theoretical predictions for the top-anti top production in hadronic collisions is shortly reviewed, paying a particular attention to the analytic calculation of the two-loop QCD corrections to the parton-level matrix elements.

35th International Conference of High Energy Physics - ICHEP2010,

July 22-28, 2010

Paris France

${ }^{*}$ Speaker. 
Since its discovery in 1995, the top quark properties were extensively studied at the Fermilab Tevatron. In the last 15 years, many observables concerning top-quark physics were measured with remarkable accuracy. Among others, the $t \bar{t}$ total cross section, $\sigma_{t \bar{t}}$, was measured with an accuracy of $\Delta \sigma_{t \bar{t}} / \sigma_{t \bar{t}} \sim 9 \%$. Data coming from the LHC are expected to improve significantly the measurement of several observables related to the top quark. Already within a couple of years of data taking in the low-luminosity low-energy phase $\left(\mathscr{L} \sim 100 \mathrm{pb}^{-1} /\right.$ year at $7 \mathrm{TeV}$ of center of mass energy), tens of thousands $t \bar{t}$ events before selection will be available. Consequently, already in this first phase, the accuracy of the cross section measurement is supposed to match the one reached to date at the Tevatron. In the high-luminosity phase $\left(\mathscr{L} \sim 100 \mathrm{fb}^{-1} /\right.$ year at $14 \mathrm{TeV}$ of center of mass energy) it will be possible to reach an accuracy $\Delta \sigma_{t \bar{t}} / \sigma_{t \bar{t}} \sim 5 \%$ [1]. This accuracy in the experimental measurements motivates theorists to refine the existing predictions, both for the total top-quark pair production cross section and for the related differential distributions. In the following, we will briefly outline the research program aiming to the full calculation of the next-to-next-to-leading order (NNLO) corrections to top-pair production cross section.

The full NLO QCD corrections to the total cross section were calculated in [2] in the case of "stable" on-shell top-quarks. In [3] several differential distributions were calculated at the same accuracy level. In [4] the NLO corrections to the top-pair production were evaluated by taking into account the top-quark decay in narrow-width approximation. The resummation of soft-gluon enhanced terms near the $t \bar{t}$ production threshold is implemented at the leading [5], next-to-leading [6], and next-to-next-to-leading [7] logarithmic accuracy. Approximate NNLO formulas for the total cross section were recently obtained by several groups [8].

The calculation of the corrections to the partonic cross section beyond leading order can be split in the calculation of the real corrections, in which the final state includes extra partons in addition to the top-quark pair, and in the calculation of virtual (loop) corrections to the partonic processes already present at the tree level. A complete calculation of the NNLO corrections requires the knowledge of the two-loop matrix elements for the processes $q \bar{q} \rightarrow t \bar{t}$ (quark-annihilation channel) and $g g \rightarrow t \bar{t}$ (gluon-fusion channel), as well as the $2 \rightarrow 3$ matrix elements at the one-loop level and the $2 \rightarrow 4$ matrix elements at the tree-level [9]. Moreover, in order to be able to deal with IR singularities, a NNLO subtraction method has to be implemented [10].

From the technical point of view, the calculation of the two-loop (virtual) corrections is particularly challenging. The squared matrix element for the processes $q\left(p_{1}\right)+\bar{q}\left(p_{2}\right) \rightarrow t\left(p_{3}\right)+\bar{t}\left(p_{4}\right)$ and $g\left(p_{1}\right)+g\left(p_{2}\right) \rightarrow t\left(p_{3}\right)+\bar{t}\left(p_{4}\right)$, summed over spin and color, can be expanded in powers of the strong coupling constant $\alpha_{S}$ as follows:

$$
|\mathscr{M}|^{2}(s, t, m, \varepsilon)=16 \pi^{2} \alpha_{S}^{2}\left[\mathscr{A}_{0}+\left(\frac{\alpha_{s}}{\pi}\right) \mathscr{A}_{1}+\left(\frac{\alpha_{s}}{\pi}\right)^{2} \mathscr{A}_{2}+\mathscr{O}\left(\alpha_{s}^{3}\right)\right] .
$$

where $p_{i}^{2}=0$ for $i=1,2$ and $p_{j}^{2}=-m_{t}^{2}$ for $i=3,4$. The Mandelstam variables are defined in the usual way: $s=-\left(p_{1}+p_{2}\right)^{2}, t=-\left(p_{1}-p_{3}\right)^{2}, u=-\left(p_{1}-p_{4}\right)^{2}$. Conservation of momentum implies that $s+t+u=2 m_{t}^{2}$.

The tree-level term $\mathscr{A}_{0}$ in the r. h. s. of Eq. (1) is well known in both production channels. The $\mathscr{O}\left(\alpha_{S}\right)$ term $\mathscr{A}_{1}$ arises from the interference of one-loop diagrams with the tree-level amplitude. The $\mathscr{O}\left(\alpha_{S}^{2}\right)$ term $\mathscr{A}_{2}$ consists of two parts: the interference of two-loop diagrams with the Born amplitude and the interference of one-loop diagrams among themselves, $\mathscr{A}_{2}=\mathscr{A}_{2}^{(2 \times 0)}+\mathscr{A}_{2}^{(1 \times 1)}$. 
The latter term, $\mathscr{A}_{2}^{(1 \times 1)}$, was calculated for both channels in [11]. The first term, $\mathscr{A}_{2}^{(2 \times 0)}$, originating from the two-loop diagrams, can be decomposed according to the color and flavor structures as follows:

$$
\begin{aligned}
\mathscr{A}_{2}^{(2 \times 0) q \bar{q}}= & \left(N_{c}^{2}-1\right)\left[N_{c}^{2} A^{q \bar{q}}+B^{q \bar{q}}+\frac{C^{q \bar{q}}}{N_{c}^{2}}+N_{c} N_{l} D_{l}^{q \bar{q}}+\frac{N_{l}}{N_{c}} E_{l}^{q \bar{q}}+N_{c} N_{h} D_{h}^{q \bar{q}}+\frac{N_{h}}{N_{c}} E_{h}^{q \bar{q}}+N_{l}^{2} F_{l}^{q \bar{q}}\right. \\
& \left.+N_{l} N_{h} F_{l h}^{q \bar{q}}+N_{h}^{2} F_{h}^{q \bar{q}}\right], \\
\mathscr{A}_{2}^{(2 \times 0) g g}= & \left(N_{c}^{2}-1\right)\left[N_{c}^{3} A^{g g}+N_{c} B^{g g}+\frac{1}{N_{c}} C^{g g}+\frac{1}{N_{c}^{3}} D^{g g}+N_{c}^{2} N_{l} E_{l}^{g g}+N_{c}^{2} N_{h} E_{h}^{g g}+N_{l} F_{l}^{g g}+N_{h} F_{h}^{g g}\right. \\
& \left.+\frac{N_{l}}{N_{c}^{2}} G_{l}^{g g}+\frac{N_{h}}{N_{c}^{2}} G_{h}^{g g}+N_{c}\left(N_{l}^{2} H_{l}^{g g}+N_{h}^{2} H_{h}^{g g}+N_{l} N_{h} H_{l h}^{g g}\right)+\frac{N_{l}^{2}}{N_{c}} I_{l}^{g g}+\frac{N_{h}^{2}}{N_{c}} I_{h}^{g g}+\frac{N_{l} N_{h}}{N_{c}} I_{l h}^{g g}\right],(2)
\end{aligned}
$$

where $N_{h}$ and $N_{l}$ are the number of heavy-quark flavors (in our case, only the top quark is considered heavy) and light-quark flavors, respectively. The coefficients $A, B, \ldots, I_{l h}$ in both channels are functions of $s, t$, and $m_{t}$, as well as of the dimensional regulator $\varepsilon$. These quantities were calculated in [12] in the approximation $s,|t|,|u| \gg m_{t}^{2}$. However, the results in [12] are not sufficient to obtain accurate NNLO predictions, since a large fraction of the events are characterised by values of the partonic center of mass energy which do not satisfy the ultra-relativistic limit. The complete top mass dependence of the color coefficients $A, B, \ldots$ is required. A numerical calculation of $\mathscr{A}_{2}^{(2 \times 0) q \bar{q}}$, exact in $s, t$, and $m_{t}$, was presented in [13]. Analytic expressions for all of the IR poles in $\mathscr{A}_{2}^{(2 \times 0) q \bar{q}}$ and $\mathscr{A}_{2}^{(2 \times 0) g g}$ are also available [15]; they were calculated by employing the expression of the IR divergences in a generic two-loop QCD amplitude with both massive and massless particles, derived in [14].

The analytic calculation of the coefficients $A^{q \bar{q}}, D_{l}^{q \bar{q}}, \ldots, F_{h}^{q \bar{q}}$ which appear in the quark annihilation channel, as well as of the coefficient $A^{g g}$ for the gluon fusion channel, was carried out in [16]. It must be observed that the coefficients with a $l$ or $h$ subscript receive contributions only from diagrams involving a closed light or heavy fermion loop, respectively. The leading color coefficients in the two production channels, $A^{q \bar{q}}$ and $A^{g g}$, involve planar diagrams only. The results reported in [16] were obtained by employing the Laporta Algorithm [17], implemented in the C++ code REDUZE $2[18,19]$, for the reduction to the Master Integrals [20, 16]. Subsequently, the master integrals were evaluated by means of the differential equation method [21]. The analytic expression of the master integrals can be written in terms of one- and two-dimensional harmonic polylogarithms [22]. The analytic results were evaluated numerically by employing codes which make use of a GiNaC package for the evaluation of generalized polylogarithms [23]. In [16] the analytic results were also expanded in the $s \gg m_{t}^{2}$ limit, in order to reproduce the results already obtained in [12]. Starting from the exact results of [16], it was also possible to obtain new analytic formulas which are valid in the production threshold limit $s \rightarrow 4 m_{t}^{2}$.

A complete numerical result for the two-loop corrections in the gluon fusion channel is still missing, and to date only the coefficient $A^{g g}$ was evaluated. Among the remaining color coefficients in Eq. (2), $E_{l}^{g g}, F_{l}^{g g}, G_{l}^{g g}, H_{l}^{g g}, H_{h}^{g g}, H_{l h}^{g g}, I_{l}^{g g}, I_{h}^{g g}, I_{l h}^{g g}$ can be calculated using the same technique already employed in [16]; their evaluation is in progress [24]. The remaining color coefficients both in the quark-antiquark channel $\left(B^{q \bar{q}}\right.$ and $\left.C^{q \bar{q}}\right)$ and in the gluon fusion channel $\left(B^{g g}, C^{g g}, D^{g g}, E_{h}^{g g}\right.$, $F_{h}^{g g}$, and $G_{h}^{g g}$ ) contain either crossed box topologies, or complicated massive sub-topologies. In the 
first case, the calculation of the color coefficients in a closed functional form using the differential equation method is very difficult, because of the large number of master integrals that occurs for some specific topologies. In the second case, the problems arise from the fact that it is known that already the sunrise diagram with three virtual propagators of mass $m$ and an external momentum $p$ such that $p^{2} \neq m^{2}$ can be expressed analytically only in terms of elliptic integrals [25]. This threedenominator diagram appears as a sub-topology in the color coefficients with subscript $h$. A viable solution for both issues is the semi-numerical approach adopted, for instance, for the two-loop equal-mass sunrise diagram [26].

To conclude, the calculation of the two-loop corrections to the top-quark pair production is an essential step needed for the full evaluation of the NNLO corrections to the top-quark production cross section and differential distributions. In the last few years several results were obtained for the quark annihilation channel. The calculation of the two-loop corrections in the gluon fusion channel is technically more complicated because of the larger number of diagrams involved and because the functional basis of the harmonic polylogarithms is known to be insufficient to obtain a full analytic result. However, the calculation of a large number of the color coefficients can be carried out with available methods and is in progress. The calculation of the color coefficients for which standard analytic techniques are insufficient can in principle be carried out with semianalytic methods already applied to other related problems.

Acknowledgments. The work of R. B. is supported by the Theory-LHC-France initiative of CNRS/IN2P3, T. G. and A. v.M. are supported by the Schweizer Nationalfonds (grants 200020126691 and 200020-124773). The work of C. S. was supported by the Deutsche Forschungsgemeinschaft (DFG SCHR 993/2-1).

\section{References}

[1] W. Bernreuther, J. Phys. G 35 (2008) 083001; arXiv:1008.3819; R. Frederix, arXiv:1009.6199.

[2] P. Nason et al. Nucl. Phys. B 303 (1988) 607; W. Beenakker et al. Phys. Rev. D 40 (1989) 54; Nucl. Phys. B 351 (1991) 507; M. Czakon and A. Mitov, Nucl. Phys. B 824 (2010) 111.

[3] P. Nason et al. Nucl. Phys. B 327 (1989) 49 [Erratum-ibid. B 335 (1990) 260]; M. L. Mangano et al. Nucl. Phys. B 373 (1992) 295; S. Frixione et al. Phys. Lett. B351 (1995) 555.

[4] W. Bernreuther et al. Nucl. Phys. B 690 (2004) 81; K. Melnikov and M. Schulze, JHEP 0908 (2009) 049; W. Bernreuther and Z. G. Si, Nucl. Phys. B 837 (2010) 90.

[5] E. Laenen et al. Nucl. Phys. B 369 (1992) 543; Phys. Lett. B 321 (1994) 254; E. L. Berger and H. Contopanagos, Phys. Lett. B 361 (1995) 115; Phys. Rev. D 54 (1996) 3085; Phys. Rev. D 57 (1998) 253; S. Catani et al. Phys. Lett. B 378 (1996) 329; Nucl. Phys. B 478 (1996) 273.

[6] N. Kidonakis and G. Sterman, Phys. Lett. B 387 (1996) 867; Nucl. Phys. B 505 (1997) 321; R. Bonciani et al. Nucl. Phys. B 529 (1998) 424 [Erratum-ibid. B 803 (2008) 234]; Phys. Lett. B 575 (2003) 268.

[7] M. Beneke et al. Nucl. Phys. B 828 (2010) 69; M. Czakon et al. Phys. Rev. D 80 (2009) 074017; V. Ahrens et al. JHEP 1009 (2010) 097; arXiv:1006.4682. N. Kidonakis, arXiv:1009.4935.

[8] U. Langenfeld et al. Phys. Rev. D 80 (2009) 054009; M. Cacciari et al. JHEP 0809 (2008) 127; N. Kidonakis and R. Vogt, Phys. Rev. D 78 (2008) 074005. 
[9] S. Dittmaier et al. Phys. Rev. Lett. 98 (2007) 262002; Eur. Phys. J. C 59 (2009) 625; G. Bevilacqua et al. Phys. Rev. Lett. 104 (2010) 162002; K. Melnikov, M. Schulze, Nucl. Phys. B840 (2010) 129.

[10] D.A. Kosower, Phys. Rev. D 67 (2003) 116003. A. Daleo, T. Gehrmann and D. Maître, JHEP 0704 (2007) 016; A. Gehrmann-De Ridder and M. Ritzmann, JHEP 0907 (2009) 041; A. Gehrmann-De Ridder et al. JHEP 0509 (2005) 056; E. W. N. Glover and J. Pires, JHEP 1006 (2010) 096; R. Boughezal et al. PoS RADCOR2009 (2010) 052; S. Catani and M. Grazzini, Phys. Rev. Lett. 98 (2007) 222002; M. Czakon, Phys. Lett. B693 (2010) 259; C. Anastasiou, F. Herzog and A. Lazopoulos, arXiv:1011.4867 [hep-ph].

[11] J. G. Korner et al. Phys. Rev. D 77 (2008) 094011; C. Anastasiou and S. M. Aybat, Phys. Rev. D 78 (2008) 114006; B. Kniehl et al. Phys. Rev. D 78 (2008) 094013.

[12] M. Czakon et al. Phys. Lett. B 651 (2007) 147; Nucl. Phys. B 798 (2008) 210.

[13] M. Czakon, Phys. Lett. B 664 (2008) 307.

[14] T. Becher and M. Neubert, Phys. Rev. Lett. 102 (2009) 162001; JHEP 0906 (2009) 081; Phys. Rev. D 79 (2009) 125004; A. Mitov et al. Phys. Rev. D 79 (2009) 094015; A. Ferroglia et al. Phys. Rev. Lett. 103 (2009) 201601; E. Gardi and L. Magnea, Nuovo Cim. C 32N5-6 (2009) 137;

[15] A. Ferroglia et al. JHEP 0911 (2009) 062.

[16] R. Bonciani et al. JHEP 0807 (2008) 129; R. Bonciani et al. JHEP 0908 (2009) 067; R. Bonciani et al. arXiv:1011.6661 [hep-ph].

[17] S. Laporta, Int. J. Mod. Phys. A 15 (2000) 5087; F.V. Tkachov, Phys. Lett. B 100 (1981) 65; K.G. Chetyrkin and F.V. Tkachov, Nucl. Phys. B 192 (1981) 159.

[18] A. von Manteuffel and C. Studerus, Reduze 2, to be published.

[19] C. Studerus, Comput. Phys. Commun. 181 (2010) 1293.

[20] M. Argeri et al. Nucl. Phys. B 631 (2002) 388; R. Bonciani et al. Nucl. Phys. B 661 (2003) 289 [Erratum-ibid. B 702 (2004) 359]; Nucl. Phys. B 690 (2004) 138; Nucl. Phys. B 676 (2004) 399; J. Fleischer et al. Nucl. Phys. B 547 (1999) 343; U. Aglietti and R. Bonciani, Nucl. Phys. B 668 (2003) 3; Nucl. Phys. B 698 (2004) 277; A.I. Davydychev and M.Y. Kalmykov, Nucl. Phys. B 699 (2004) 3. M. Czakon et al. Phys. Rev. D 71 (2005) 073009; G. Bell, arXiv:0705.3133; R. Bonciani and A. Ferroglia, JHEP 0811 (2008) 065.

[21] A.V. Kotikov, Phys. Lett. B 254 (1991) 158; Phys. Lett. B 259 (1991) 314; Phys. Lett. B 267 (1991) 123; E. Remiddi, Nuovo Cim. A 110 (1997) 1435.

[22] A B. Goncharov, Math. Res. Lett. 5 (1998), 497; E. Remiddi and J.A.M. Vermaseren, Int. J. Mod. Phys. A 15 (2000) 725; T. Gehrmann and E. Remiddi, Nucl. Phys. B 601 (2001) 248; Nucl. Phys. B 601 (2001) 287; Comput. Phys. Commun. 141 (2001) 296; Comput. Phys. Commun. 144 (2002) 200; D. Maître, Comput. Phys. Commun. 174 (2006) 222; hep-ph/0703052.

[23] J. Vollinga and S. Weinzierl, Comput. Phys. Commun. 167 (2005) 177.

[24] R. Bonciani, A. Ferroglia, T. Gehrmann, A. von Manteuffel, and C. Studerus, in preparation.

[25] S. Laporta, E. Remiddi, Nucl. Phys. B704, 349-386 (2005).

[26] S. Pozzorini and E. Remiddi, Comput. Phys. Commun. 175 (2006) 381; U. Aglietti et al. Nucl. Phys. B 789 (2008) 45. 